\title{
Mission to Mars to Collect a Storehouse of Scientific Data
}

PAGES 441-442

\section{Arden Albee}

Just after Election Day, the Mars Global Surveyor spacecraft (Figure 1) will embark on a journey to Mars to examine the surface and the seasonal variations of the atmosphere over an entire Mars year. Mars is an extremely rich mission target because the scientific questions it poses touch on geology, geophysics, geochemistry, atmospheric physics, climatology, biology, and-most of all-comparative planetology.

Researchers will use data collected from this mission to compare the planet's formation and evolution with that of Earth, to understand the evolution of climate and water on Mars, and to determine if life has ever existed on Mars. Mars Global Surveyor will acquire a basic global understanding of the planet as it exists today, provide a framework for understanding its past, and help shape a series of small missions to Mars that are being planned for the next 10 years.

This mission addresses most of the objectives of the Mars Observer Mission, which was lost in August 1993, just prior to entering orbit at Mars. But Mars Global Surveyor has cost much less; the spacecraft has been built in less time; and it is to be launched on a smaller launch vehicle. There are some other differences. The scientific instruments on Mars Global Surveyor are either spares or duplicates of the Mars Observer instruments, but the two heaviest instruments from the Mars Observer could not be included. Also, the limited capability of the launch vehicle does not permit an all-propulsive transfer into the desired circular orbit at Mars. Instead, this orbit will be achieved by aerobraking - by dipping repeatedly-into the planet's upper atmosphere to slow the spacecraft and reduce the size of the spacecraft's orbit.

The Mars Global Surveyor will be launched from Cape Canaveral on Novem-

California Institute of Technology, Division of Geological \& Planetary Science, MS 170-25, Pasadena, CA 91125 ber 6 aboard a Delta II rocket. The three-axis stabilized spacecraft uses most of the spare components from the Mars Observer spacecraft in a configuration that is about half the

size of the larger craft's. Two solar arrays, each $3.5 \times 1.9 \mathrm{~m}$, provide $980 \mathrm{~W}$ of power, and the back side of the panels is used to provide drag for the aerobraking maneuvers. No movable scan platform is provided; the spacecraft will be pointed toward nadir as it maps Mars, rotating at the orbital rate, with the antenna tracking Earth and the solar arrays tracking the Sun.

Instrument checkout and calibrations will be performed a few days after launch, in part using Earth as the target, and calibrations will continue through the 10-month trip to Mars. Figure 2 shows the mission profile and the various configurations of the spacecraft dur-

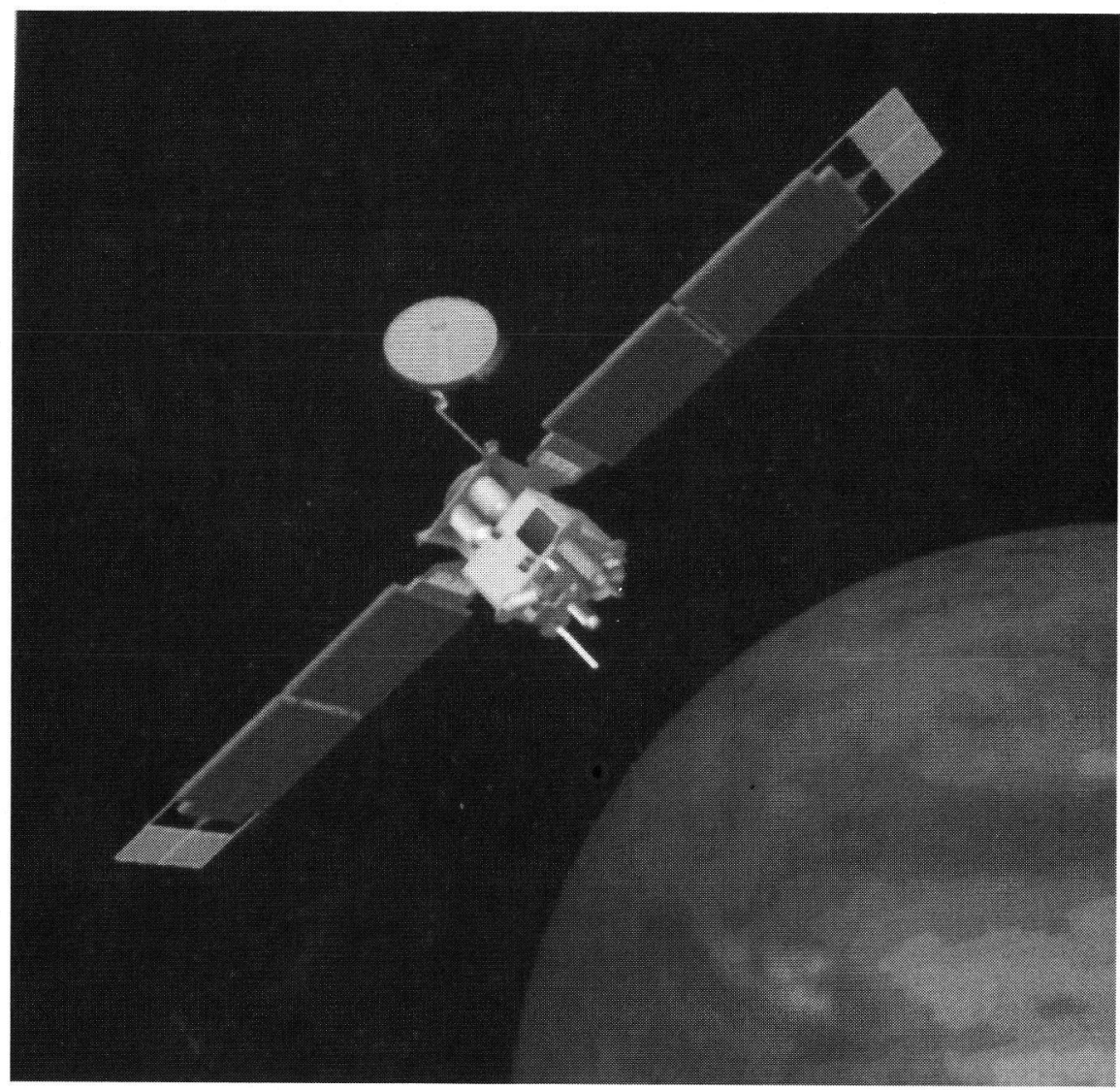

Fig. 1. The Mars Global Surveyor spacecraft and its payload in the fully deployed configuration of its mapping orbit at Mars. The instruments on the nadir panel of the spacecraft point to the surface, the antenna on its boom tracks Earth, and the articulated solar panels track the Sun as the spacecraft orbits Mars. The tall, fat cylinder contains the three line-scan cameras, and the antenna that will relay data from landers on the surface back to Earth is the tall slender cylinder. The low cylinder next to the line-scan cameras is the laser altimeter mirror. On the other side of the line-scan cameras is the thermal emission spectrometer. The magnetometer sensors are located on the triangular tabs at the end of each solar panel. The flap on the end of each solar panel provides additional resistance during aerobraking. Original color image appears at the back of this volume. 


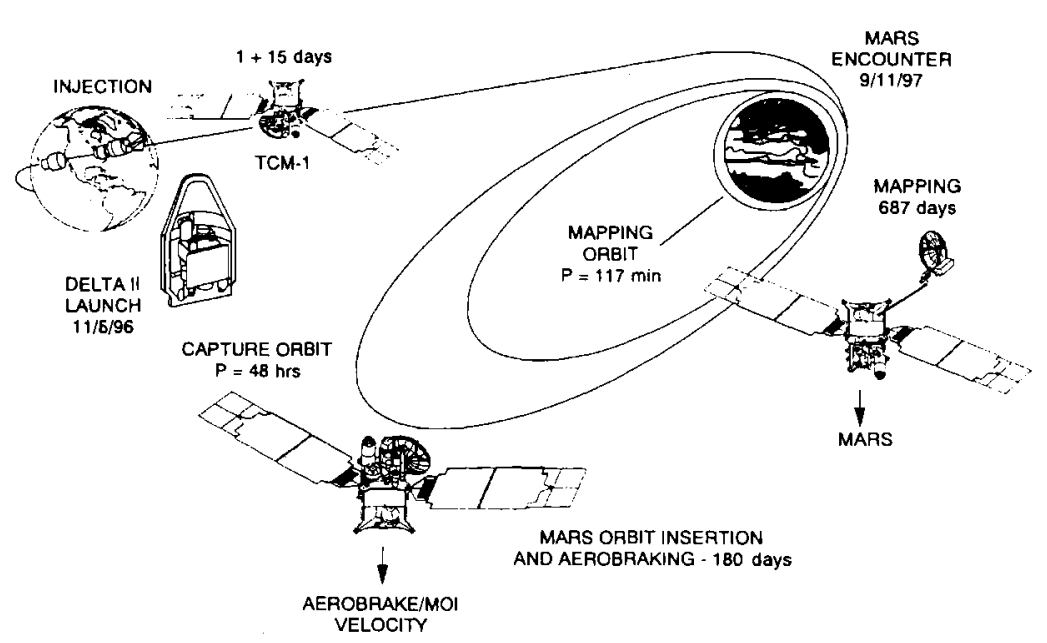

Fig. 2. Mission profile for the Mars Global Surveyor mission illustrating the spacecraft configuration during each phase.

ing the mission. The main engine will be fired to place the spacecraft into a highly elliptical capture orbit. Over the following 6 months, aerobraking maneuvers and thrusters will gradually change the orbit into a near-circular, sun-synchronous, low-altitude mapping orbit. Some measurements will be obtained during the early part of the aerobraking period, perhaps including observations of Phobos, and data will be relayed from the Russian landers on the Martian surface. The operational complexities of the aerobraking maneuvers, however, will prevent observations during most of the aerobraking transition to the mapping orbit. The planet will be mapped repeatedly in 26-day cycles with a constant sun angle, allowing differentiation between surface characteristics that vary daily from those due to seasonal changes.

Mapping data collected by the instruments will be continuously recorded on the solid-state recorder during the 687-day mapping period from March 1998 to February 2000. Twenty-four hours of recorded science data will be returned to Earth each day during a single 10-hour session with the tracking stations of the Deep Space Network.

The Mars Global Surveyor instruments include three line-scan cameras; a thermal emission spectrometer; a laser altimeter that uses the spacecraft radio system with an ultrastable oscillator; magnetometers complemented with an electron reflectometer; and a radio system to relay data from landers on the surface of Mars. Detailed descriptions of the instruments and the experiments were published in 1992 in a Mars Observer Spe- cial Issue of the Journal of Geophysical Research.

The three line-scan cameras will photograph portions of Mars at unprecedented resolution, looking at the surface effects of wind, water, ice, volcanic eruption, earthquake faulting, landsliding, and other geological processes. Features as small as $1.4 \mathrm{~m}$ will be seen and those 4-7 $\mathrm{m}$ across will be recognizable. The line-scan cameras will also photograph the entire planet regularly in two colors at lower resolution to provide timelapse coverage of the surface and atmosphere, much like Earth-orbiting weather satellites do

The thermal emission spectrometer uses a Michelson interferometer that measures the emitted infrared spectrum to determine the mineral composition of Martian rocks and soils and the composition of ices, atmospheric dust, and clouds. Identifying and finding carbonate minerals will be especially important for choosing sites for future lander missions that will search for evidence of life on Mars. Detecting water-bearing minerals will provide information on the presence of water throughout Martian history. The thermal emission spectrometer will also measure temperature and pressure in the atmosphere, providing clues to its circulation, which will in turn be used to infer possible climate change. It will also return valuable information on the energy balance of the Martian surface and atmosphere.

The laser altimeter maps the topography of Mars using the travel time of a laser pulse from the spacecraft to the surface and back.
The formation processes of Martian volcanoes, impact structures, channels and canyons, and the volume of polar ice will be studied. The topographic data will be used with gravity data from the spacecraft radio system and ultrastable oscillator experiment to infer the structure and temperature of the Martian interior, thereby providing important information about how the interior evolved. The radio signal from the spacecraft passes through the Mars atmosphere on its way to Earth twice during each orbit. The radio signal is modified by the atmosphere and provides high vertical resolution profiles of the physical properties of the atmosphere.

Another investigation, using magnetometers complemented with an electron reflectometer, will establish the existence and strength of the Martian magnetic field. Detection of a magnetic field will provide direct evidence for the past or present existence of active motions in a fluid core. The electron reflectometer will measure remnant crustal magnetization that will be used to examine changes in the magnetic field over geologic time. These observations will provide powerful insights into the composition, thermal state, evolution, and dynamics of the interior.

Mars Relay provides a communication link to surface landers sent to Mars by the Russian Mars 1996 and the U.S. Mars 1998 missions. This link to the Mars Global Surveyor will increase the amount of data these landers can return to Earth, greatly enhancing the scientific return from such missions.

Collectively, the instruments on the Mars Global Surveyor form a highly complementary set; each produces a well-defined set of measurements that address specific objectives, but each data set also contributes to a much wider variety of scientific investigations. The data set surpasses in volume the data returned by all the Mariner, Viking, and Voyager missions put together, and its archive will provide a rich storehouse for investigators for many decades to come.

Images of spacecraft and instrument assembly, illustrative charts, and additional information on the instruments and the mission can be obtained by Internet (http://mgs-www.jpl.nasa.gov).

\section{Science Team Leaders}

M. Acuna, A. Albee, R. Arvidson, J. Blamont, M. Carr, P. Christensen, R. Haberle, A. Ingersoll, B. Jakosky, M. Malin, D. Smith, L. Soderblom, and G. L. Tyler 
Vol. 77, No. 45, Novernber 5, 1996

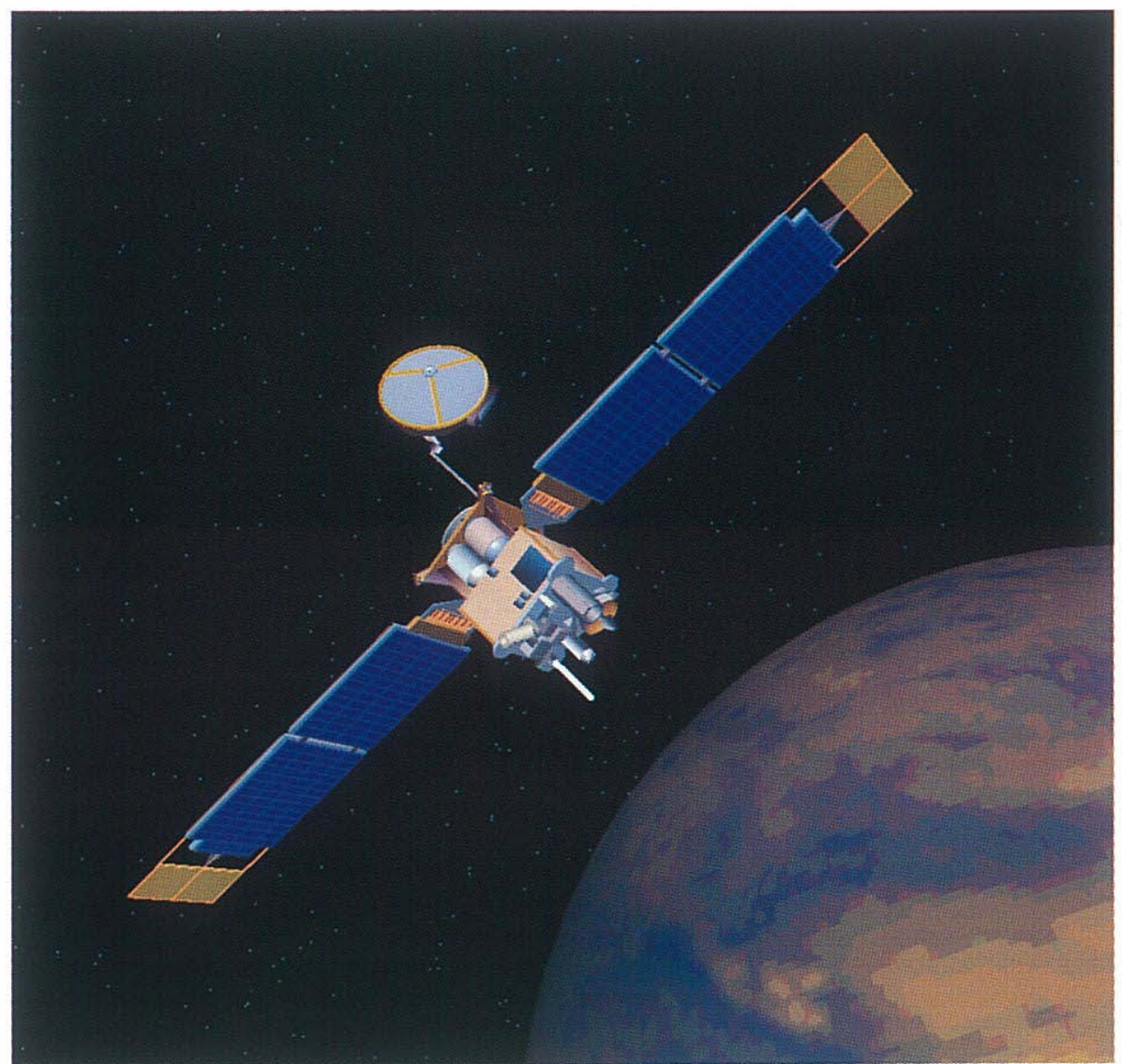

Fig. I. The Mars Global Surveyor spacecraft and its payload in the fully deployed configuration of its mapping orbit at Mars. The instruments on the nadir panel of the spacecraft point to the surface, the antenna on its boom tracks Earth, and the articulated solarpanels track the Sun as the spacecraft orbits Mars. The tall, fat cylinder contains the three line-scan cameras, and the antenna that will relay data from landers on the surface back to Earth is the tall slender cylinder. The low cylinder next to the line-scan cameras is the laser altimeter mirror. On the other side of the line-scan cameras is the thermal emission spectrometer. The magnetometer sensors are lo cated on the triangular tabs at the end of each solar panel. The flap on the end of each solar panel provides additional resistance during aerobraking. 